УДК 536.25

\title{
Numerical Investigation of a Dependence of the Dynamic Contact Angle on the Contact Point Velocity in a Problem of the Convective Fluid Flow
}

\author{
Olga N. Goncharova* \\ Altai State University \\ Lenina, 61, Barnaul, 656049 \\ Russian
}

Alla V. Zakurdaeva

Institute of Thermophysics SB RAS

Lavrentyev, 1, Novosibirsk, 630090

Russian

Received 07.12.2015, received in revised form 09.02.2016, accepted 20.06.2016

\begin{abstract}
A two-dimensional problem of the fluid flows with a dynamic contact angle is studied in the case of an uniformly moving contact point. Mathematical modeling of the flows is carried out with the help of the Oberbeck-Boussinesq approximation of the Navier-Stokes equations. On the thermocapillary free boundary the kinematic, dynamic conditions and the heat exchange condition of third order are fulfilled. The slip conditions (conditions of proportionality of the tangential stresses to the difference of the tangential velocities of liquid and wall) are prescribed on the solid boundaries of the channel supporting by constant temperature. The dependence of the dynamic contact angle on the contact point velocity is investigated numerically. The results demonstrate the contact angle behavior and the different flow characteristics with respect to the various values of the contact point velocity, friction coefficients, gravity acceleration and an intensity of the thermal boundary regimes.
\end{abstract}

Keywords: convective flow, free boundary, dynamic contact angle, moving contact point, mathematical model, computational algorithm.

DOI: 10.17516/1997-1397-2016-9-3-296-306.

\section{Introduction}

The problems of flows of a viscous incompressible fluid in the domains with interfaces are very important for investigations. The features of the fluid flows in the domains with free boundaries and interfaces are the subject of many investigations in the last decade. Such interest is explained by need of study some phenomena in the flow structure, which arise due to the effects related with the gas phase and solid wall properties. One of the most important questions of mathematical modeling of the convective fluid flows in a domain with an interface is a correct formulation of the boundary conditions. From the mathematical point of view the non-stationary fluid flows with free boundaries remain to be very difficult for investigations because of the dynamic contact angle problem [1-6]. The problem of dynamic contact angle occurs due to the incompatibility of the conditions on the free surface of the liquid and the conditions of adhesion on a solid surface

*gon@math.asu.ru

(c) Siberian Federal University. All rights reserved 
in vicinity of the moving three-phase contact line. There are various methods of statement of the problems with contact angles, describing a motion of a viscous incompressible liquid in the presence of a moving contact line (or contact point in the two-dimensional case). These problem statements assume a replacement of the no-slip conditions terms by the slip conditions on some sections of the solid walls near the contact line, the asymptotic approach, the assumption of the contact angle equality to $\pi$ or to "zero" etc. (see, for example, $[1,2,7,8]$ ). For some mathematical models of fluid flows with dynamic contact angle the correctness of the initial boundary-value problems has been proved $[1,2,7,9-11]$.

The problem of the fluid flows in a two dimensional domain will be studied in the case when the contact points are moving with a constant velocity. A behavior of the contact angle depends on the velocity of movement of the contact point, on the nature of the boundary thermal conditions specified on solid walls and free thermocapillary boundary, on the values of the friction coefficients and also on intensity of the gravitation field. We consider the Oberbeck-Boussinesq equations of convection. The Cartesian coordinate system is chosen in such a way that the gravity acceleration vector is directed along the longitudinal axis. The problem is studied in a quasi-stationary formulation as a result of the introduction of the coordinate system moving with the liquid.

We investigate numerically the dependence of the contact angle on the contact point velocity in the case of different values of the friction coefficients and effects of the thermal boundary condition, prescribed on the free surface, on the found dependence.

\section{Governing equation}

We study the two-dimensional problem with dynamic contact angle and present the problem statement written in the coordinate system moving together with the liquid. The linear size $l$ of the flow domains $\Omega$ in the $y$-direction has been chosen for the characteristic length. The characteristic values for the problem of the fluid flow in $\Omega$ are: $u_{*}$ is the characteristic velocity, $p_{*}=\rho u_{*} \nu / l$ is the characteristic pressure, $T_{*}$ is the characteristic temperature (characteristic temperature drop). The values of the characteristic scales are specified below (see Section 3 ).

Let $\Omega=\left\{(x, y) \mid y \in(0,1), f(y)<x<x_{0}\right\}$ be the flow domain (see Fig.1). The boundary $\partial \Omega$ of the flow region contains the solid parts $\Gamma_{0}=\left\{(x, y) \mid y \in(0,1), x=x_{0}\right\}, \Gamma_{s}=\{(x, y) \mid y \in$ $\left.\{0,1\}, x \in\left(0, x_{0}\right)\right\}$ and free unknown boundary $\Gamma_{f}=\{(x, y) \mid y \in(0,1), x=f(y)\}$. The gravity acceleration vector is $\mathbf{g}=(g, 0)$. The Oberbeck-Boussinesq approximation of the Navier-Stokes equations $[12,13]$ is used to model the liquid flows and to find the unknown functions such as stream function $\psi$, vorticity $\omega$ and temperature $T$ :

$$
\begin{gathered}
\Delta \psi=-\omega, \\
\operatorname{Re}\left(u \omega_{x}+v \omega_{y}\right)=\Delta \omega+\frac{G r}{R e} T_{y}, \\
\operatorname{Re} \operatorname{Pr}\left(u T_{x}+v T_{y}\right)=\Delta T .
\end{gathered}
$$

Here

$$
u=\psi_{y}, \quad v=-\psi_{x}, \quad \omega=v_{x}-u_{y}
$$

$\mathbf{v}=(u, v)$ is the velocity vector.

Formulation of the conditions on the free boundary in terms of the stream function and vorticity has been also made in $[1,14-16]$. We generalize the results from $[8]$ for the case of the 


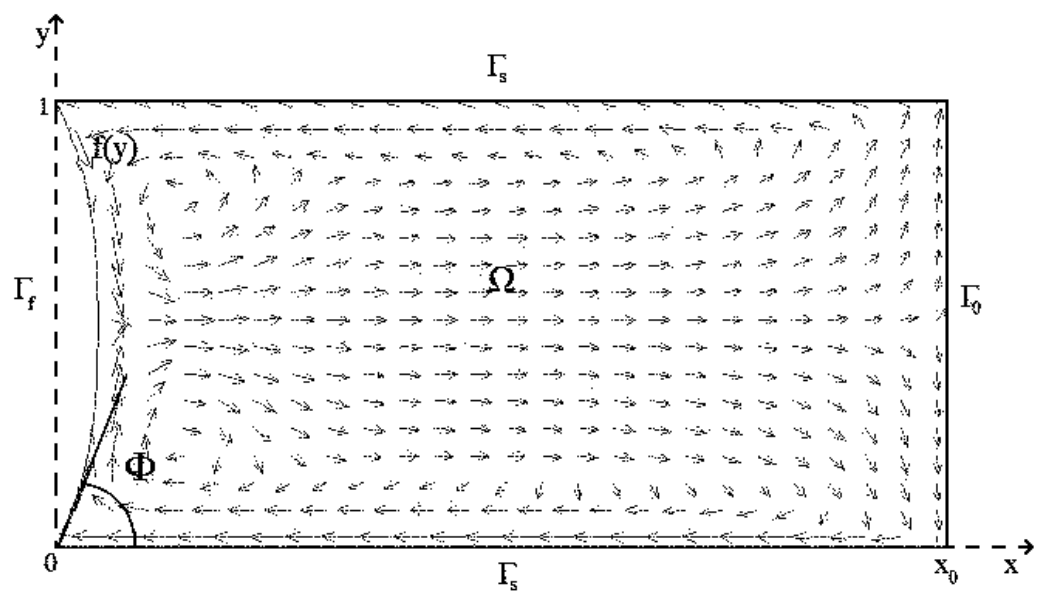

Fig. 1. Geometry of flow region; topology of flow

convective fluid flows in the domain $\Omega$ with the thermocapillary boundary $\Gamma_{f}$. Direct calculations lead to the following results:

$$
\begin{gathered}
\psi=0 \text { on } \partial \Omega, \\
\psi_{n n}-H \psi_{n}=-M a T_{\tau}, \text { on } \Gamma_{f}, \\
\frac{1}{\sqrt{1+f^{\prime 2}}}\left(-\bar{\sigma} \sigma H^{\prime}+M a T_{\tau} H+\bar{g} f^{\prime}\right)=Q(\psi) \text { on } \Gamma_{f}, \\
Q(\psi)=R e\left(\psi_{y}\left(\psi_{x}\right)_{n}-\psi_{x}\left(\psi_{y}\right)_{n}\right)-(\Delta \psi)_{n}-2\left(\psi_{n}\right)_{\tau \tau} . \\
\bar{\alpha} \psi_{n n}+\psi_{n}=-S \text { on } \Gamma_{s}(y=0), \\
\bar{\alpha} \psi_{n n}+\psi_{n}=S \text { on } \Gamma_{s}(y=1), \\
\bar{\alpha} \psi_{n n}+\bar{\gamma} \psi_{n}=0 \text { on } \Gamma_{0}\left(x=x_{0}\right), \\
T_{n}=-N u\left(T-T_{e x}\right), \text { on } \Gamma_{f}, \\
T=T_{s}, \text { on } \Gamma_{s}(y=0) \text { and }(y=1), \\
T=T_{b}, \quad \text { on } \Gamma_{0}\left(x=x_{0}\right) .
\end{gathered}
$$

At the contact points we have the conditions

$$
f(0)=f(1)=0 .
$$

The contact angle $\Phi$ at $y=0$ is computed as $\Phi=\arccos \left(f^{\prime}(0) / \sqrt{1+f^{\prime 2}(0)}\right)$.

The derivatives $(\partial / \partial n)$ and $(\partial / \partial \tau)$ with respect to the normal and tangential directions are calculated according to the rules $\partial / \partial n=\mathbf{n} \cdot \nabla, \partial / \partial \tau=\tau \cdot \nabla$. Here $\mathbf{n}$ is the external normal vector with respect to $\Omega, \tau$ is the tangential vector to the boundary $\partial \Omega$ (transition from $\tau$ to the vector $\mathbf{n}$ is in anticlockwise direction). The boundary conditions (5)-(11) are a sequence of the nonpermeability condition including the kinematic condition at free boundary (see (5)), the slip conditions which follow from the assumption of the impulse conservation (see (9)-(11)), the dynamic conditions on free surface (projections of the stress vector on the tangential (6) and normal (7) vectors to $\Gamma_{f}$ ). The third order condition for temperature (12) due to the Newton 
law of heat transfer is assumed to be fulfilled on the free boundary. The temperature on the lateral solid walls is kept constant equal to $T_{s}$ and $T_{b}$ (see (13), (14)). The boundary conditions for temperature (12)-(14) allow to stick the consideration of the symmetric flow picture.

The following nondimensional parameters appear: $R e=v_{*} l / \nu$ is the Reynolds number, $\operatorname{Pr}=\nu / \chi$ is the Prandtl number, $M a=\sigma_{T} T_{*} /\left(\rho \nu v_{*}\right)$ is the Marangoni number, $C a=\rho \nu v_{*} /\left(\sigma_{0}\right)$ is the capillary number, $G r=\beta T_{*} g l^{3} / \nu^{2}$ is the Grashof number, $N u=\delta l / \kappa$ is the Nusselt number, $\bar{\alpha}=\rho \nu /(\gamma l), \bar{\gamma}=\gamma_{0} / \gamma, \bar{\sigma}=\sigma_{0} /\left(\rho \nu v_{*}\right)=1 / C a, \bar{g}=g l^{2} /\left(\nu v_{*}\right)=G r /\left(\operatorname{Re} \beta T_{*}\right)$. Here $\sigma$ is the nondimensional surface tension $\left(\sigma=1-\operatorname{MaCa}\left(T-T_{0}\right)\right), \nu$ and $\chi$ are the coefficients of kinematic viscosity and thermal diffusivity, respectively, $\kappa$ is the thermal conductivity, $\rho$ is the density of the liquid, $\beta$ is the thermal expansion coefficient, $\gamma, \gamma_{0}$ are the friction coefficients of the liquid on the solid walls, $\sigma_{0}$ is the surface tension value (dimensional) at some reference temperature, $\sigma_{T}$ is the temperature coefficient of surface tension, $\delta$ is the inter-phase heat transfer coefficient, $H=\left(f^{\prime} / \sqrt{1+f^{\prime 2}}\right)^{\prime}$ is the curvature of the boundary $\Gamma_{f}$ (here "prime" denotes derivative with respect to $y$ ), $T_{e x}$ is the external temperature (non-dimensional).

To solve the problem (1)-(15) in the fixed domain $\bar{\Omega}=\{[0,1] \times[0,1]\}$ we introduce the new spatial variables

$$
z=\frac{x-f(y)}{x_{0}-f(y)}, y=y .
$$

Introduction of the variables (16) demand to recalculate the derivatives in the equations (1)-(4) and in the conditions (6)-(12).

Then the equations (1), (2) and (3) can be rewritten in new variables (16) in the following general form:

$$
\frac{1}{B_{11}}\left(U_{y}+V_{z}\right)+G=0
$$

Here

$$
U=B_{11} \Psi_{y}+B_{12} \Psi_{z}+B \Psi \psi_{z}, \quad V=B_{12} \Psi_{y}+B_{22} \Psi_{z}-B \Psi \psi_{y},
$$

the coefficients $B_{11}, B_{12}, B_{22}$ and $B$ and the functions $\Psi, G$ are specified as follows:

$$
B_{11}=x_{0}-f(y), \quad B_{12}=(z-1) f^{\prime}(y), \quad B_{22}=\frac{1+(z-1)^{2} f^{\prime 2}(y)}{x_{0}-f(y)},
$$

$G=\omega, B=1$ if $\Psi=\psi ; G=-(G r / R e)\left(\left(f^{\prime}(z-1) /\left(x_{0}-f\right)\right) T_{z}+T_{y}\right), B=\operatorname{Re}$ if $\Psi=\omega ; G=0$, $B=\operatorname{Re} \operatorname{Pr}$ if $\Psi=T$.

The conditions (5)-(11) for $\psi$ and $\omega$ on the boundaries of $\bar{\Omega}$ and the boundary conditions for temperature $T$ defined by the conditions (12)-(14) can be written as follows:

$$
\begin{gathered}
\psi=0, \psi_{y}+\bar{\alpha} \omega=-S \text { on } y=0, \\
\psi=0, \psi_{y}-\bar{\alpha} \omega=-S \text { on } y=1, \\
\psi=0, \frac{2 f^{\prime \prime}}{\left(x_{0}-f\right)\left(1+f^{\prime 2}\right)} \psi_{z}+\frac{M a}{\left(x_{0}-f\right)^{2}\left(1+f^{\prime 2}\right)^{5 / 2}} T_{y}-\omega=0 \text { on } z=0, \\
\psi=0, \frac{\bar{\gamma}}{x_{0}-f} \psi_{z}-\bar{\alpha} \omega=0 \text { on } z=1, \\
T=T_{s} \text { on } y=0, \\
T=T_{s} \text { on } y=1,
\end{gathered}
$$




$$
\begin{gathered}
-\frac{\sqrt{1+f^{\prime 2}}}{x_{0}-f} T_{z}+\frac{f^{\prime}}{\sqrt{1+f^{\prime 2}}} T_{y}=-N u\left(T-T_{e x}\right) \text { on } z=0, \\
T=T_{0} \text { on } z=1 .
\end{gathered}
$$

The equation (7), written in the variables $z, y,(16)$ will be used to compute the free boundary position.

\section{Computational algorithm}

To reach the stationary solution of the problem we organize an iteration process for solving of the general equation (17):

$$
\Psi_{t}=\frac{\lambda}{B_{11}}\left(U_{y}+V_{z}\right)+\lambda G
$$

Here $\lambda$ is an iteration parameter. To discretize in time we choose a time stepsize $\Delta t$, define $t_{k}=k \Delta t(k=0,1,2, \ldots), \Psi^{k}=\Psi\left(t_{k}, \cdot\right)$. After discretization of (28) in time we introduce the spatial grids $\left(z_{n}, y_{m}\right) ; z_{n}=(n-1) h_{z}\left(n=1,2, \ldots, N_{1}\right), h_{z}=1 / N\left(N_{1}=N+1\right) ; y_{m}=(m-1) h_{y}$ $\left(m=1,2, \ldots, M_{1}\right), h_{y}=1 / M\left(M_{1}=M+1\right)$ and approximate $\Psi^{k}\left(x_{n}, y_{m}\right)$ by $\Psi_{n, m}^{k}$. The finite-difference scheme of the form

$$
\begin{gathered}
\frac{\Psi^{k+\frac{1}{2}}-\Psi^{k}}{0.5 \Delta t}=\frac{\lambda}{B_{11}}\left\{U_{y}^{k}+V_{z}^{k+1 / 2}\right\}+\lambda G^{k}, \\
\frac{\Psi^{k+1}-\Psi^{k+\frac{1}{2}}}{0.5 \Delta t}=\frac{\lambda}{B_{11}}\left\{U_{y}^{k+1}+V_{z}^{k+1 / 2}\right\}+\lambda G^{k}
\end{gathered}
$$

is used to solve (28). The derivatives in (29) will be approximated by the finite-difference analogs of second orders. We present an example for the approximation for $U_{y}^{k+1}\left(x_{n}, y_{m}\right)$ $\left(U_{y}^{k+1}\left(x_{n}, y_{m}\right)=(\partial U / \partial y)^{k+1}\left(x_{n}, y_{m}\right)\right)($ see $(18))$ :

$$
\begin{gathered}
U_{y}^{k+1}\left(x_{n}, y_{m}\right)=\frac{\partial}{\partial y}\left(B_{11} \Psi_{y}^{k+1}\right)\left(x_{n}, y_{m}\right)+\frac{\partial}{\partial y}\left(B_{12} \Psi_{z}^{k+1 / 2}\right)\left(x_{n}, y_{m}\right)+B \frac{\partial}{\partial y}\left(\Psi^{k+1} \psi_{z}\right)\left(x_{n}, y_{m}\right) \approx \\
\approx \frac{1}{h_{y}^{2}}\left(\left(B_{11}\right)_{n, m+1 / 2}\left(\Psi_{n, m+1}^{k+1}-\Psi_{n, m}^{k+1}\right)-\left(B_{11}\right)_{n, m-1 / 2}\left(\Psi_{n, m}^{k+1}-\Psi_{n, m-1}^{k+1}\right)\right)+ \\
+\frac{1}{4 h_{z} h_{y}}\left(\left(B_{12}\right)_{n, m+1}\left(\Psi_{n+1, m+1}^{k+1 / 2}-\Psi_{n-1, m+1}^{k+1 / 2}\right)-\left(B_{12}\right)_{n, m-1}\left(\Psi_{n+1, m-1}^{k+1 / 2}-\Psi_{n-1, m-1}^{k+1 / 2}\right)\right)+ \\
+\frac{B}{4 h_{z} h_{y}}\left(\Psi_{n, m+1}^{k+1}\left(\psi_{n+1, m+1}-\psi_{n-1, m+1}\right)-\Psi_{n, m-1}^{k+1}\left(\psi_{n+1, m-1}-\psi_{n+1, m-1}\right)\right) .
\end{gathered}
$$

The following relation $\left(B_{11}\right)_{n, m+1 / 2}=0.5\left(B_{11}\left(z_{n}, y_{m}\right)+B_{11}\left(z_{n}, y_{m+1}\right)\right)$ is used for the approximation of $B_{11}$ depending on $z, y$. The finite-difference approximations of the boundary conditions (20)-(27) will be added to (29) in order to obtain the equations for $n=1$ and $n=N_{1}$ $(m=2, \ldots, M)$ and also for $m=1$ and $m=M_{1}(n=2, \ldots, N)$. The finite-difference analogs for the derivatives of first order at the boundary are used. The presented longitudinal transverse finite difference scheme (29), known as the method of alternating directions [17-19], is the scheme formally of second order of approximation and unconditionally stable $[17,18,20]$. As a result two systems of the linear algebraic equations will be obtained. It will allow us to find $\Psi_{n, m}$ as the solution of these systems of linear algebraic equations, which can be stably solved by the variants of the Gaussian elimination $[8,13,21]$ or the Thomas algorithms in the $y$-and $z$-directions. 
The numerical algorithm for the problem (17)-(27) contains the important part of computation of the free boundary position. The computational algorithm for $f(y)$ (and also for $f_{0}(y)$ ) has been described in details in [8]. We limit ourselves to the following comments.

We proceed from an initial situation when for given prescribed $x_{0}$ the liquid is at some constant temperature $T_{i n}$ (for instance, $T_{i n}=T_{0}$ ) and at rest. The initial position of the free boundary will be defined as a solution of the problem: $\bar{\sigma} H_{0}^{\prime}-\bar{g} f_{0}^{\prime}=0$ in $(0,1), \mu_{0} f_{0}^{\prime}(y)=$ $\cos \left(\Phi_{0}\right)$ for $y=0$ and $\mu_{0} f_{0}^{\prime}(y)=-\cos \left(\Phi_{0}\right)$ for $y=1, f_{0}(0)=f_{0}(1)=0$,

$$
\int_{0}^{1}\left(x_{0}-f_{0}(y)\right) d y=V \text {. }
$$

Here we denoted $\mu_{0}=1 / \sqrt{1+f_{0}^{2}}$. Note, in the case of prescribed liquid volume $V$ we determine $x_{0}$ according to (30). In the further considerations we will compute the solutions (i.e. the free boundary position $f$ and all the unknown functions) for the found pair $\left(V, x_{0}\right)$.

We seek the new dependent function $w\left(w=f-f_{0}\right)$ as a solution of the problem

$$
\begin{gathered}
\left(\mu_{0} w^{\prime}\right)^{\prime \prime}-\overline{\bar{g}} w^{\prime}=\bar{Q} \text { in }(0,1), \\
w(0)=w(1)=0, \\
\int_{0}^{1} w(y) d y=0
\end{gathered}
$$

for given $S, \psi, \omega, T$ and $f_{0}$. Here $\overline{\bar{g}}=\bar{g} / \bar{\sigma}, \bar{Q}=-(1 / \bar{\sigma})\left(Q / \mu_{f}\right)-\left(f^{\prime}\left(\mu_{f}-\mu_{0}\right)\right)^{\prime \prime}+$ $\left.(M a / \bar{\sigma})\left(1 / \mu_{f}\right)\left((H T)_{\tau}\right)\right|_{\Gamma_{f}}$, see (7) and (8) for determination of $Q$. Similarly to $\mu_{0}$ we denote $\mu_{f}=1 / \sqrt{1+f^{2}}$. The term $\left.(H T)_{\tau}\right|_{\Gamma_{f}}$ is recalculated with respect to new spatial variables $(z, y)$ (see (16)) and used at $z=0$.

The approximate solution for $w$ can be constructed with the help of an uniform grid $\left(y_{1}, y_{2}, \ldots, y_{M_{1}}\right), y_{m}=(m-1) h_{y}, h_{y}=1 / M, M_{1}=M+1$. The problem has been simplified under assumption of the symmetry of the problem relative to the line $y=0.5$ [8]. The grid is introduced so that $y_{m}=0.5$ for $\bar{m}=(M / 2)+1$. As a result, after introduction of an auxiliary function $q=\mu_{0} w^{\prime}$ the problem (31)-(33) has been solved on the interval $(0,1)$ with use of the constructed variant of the Gaussian elimination procedure [8]. The assumptions of symmetry of the $w$ - function and anti-symmetry of $q$ - function $\left(q_{\bar{m}}\right)$ have been used.

The computational algorithm for $f_{0}$ has been constructed in $[8]$ with use of the procedure for $w$ by $\bar{Q}=0$. Starting with $f_{0}^{0}=0$ a sequence of iterates $\left\{f_{0}^{1}, f_{0}^{2}, \ldots\right\}$ has been generated with the help of an iteration algorithm.

\section{Numerical results}

The liquid of the volume $V$ (with the free boundary) is moving with the constant velocity in the $x$-direction. The contact angle (the angle between the solid wall and free boundary) is calculated in the position being inside the liquid. It should be noted that the contact angle dependence on the contact point velocity is characterized by increasing of the contact angle value with an increase of the velocity. The effects of the friction coefficients $\left(\gamma, \gamma_{0}\right)$ and of the gravity acceleration $(g)$ on the dependence of the contact angle $\Phi$ on the contact point velocity $S$ are investigated.

The numerical experiments are performed for the liquid being water. The physical parameters of the problem are presented in Tab. $1([22,23])$. For the characteristic velocity we choose 
Table 1. Physical parameters of the problem.

\begin{tabular}{|l|c|c|c|c|c|c|c|c|}
\hline Parameter & $\rho$ & $\nu$ & $\beta$ & $\sigma_{T}$ & $\sigma_{0}$ & $\gamma=\gamma_{0}$ & $\kappa$ & $\chi$ \\
\hline & $\frac{g}{\mathrm{~cm}^{3}}$ & $\frac{\mathrm{cm}^{2}}{\mathrm{~s}}$ & $\frac{1}{K}$ & $\frac{d y n e}{\mathrm{cmK}}$ & $\frac{d y n e}{\mathrm{~cm}}$ & $\frac{\mathrm{g}}{\mathrm{cm}^{2} \mathrm{~s}}$ & $\frac{\mathrm{kal}}{\mathrm{cmsK}}$ & $\frac{\mathrm{cm}}{\mathrm{s}}$ \\
\hline Water & 1 & 0.008 & $0.15 \cdot 10^{-3}$ & 0.1514 & 72 & $1 ; 100$ & $0.144 \cdot 10^{-2}$ & $0.14 \cdot 10^{-2}$ \\
\hline
\end{tabular}

$v_{*}=\left(\sigma_{0} g / \gamma\right)^{1 / 3}$ in the case when $g=981\left(\mathrm{~cm} / \mathrm{s}^{2}\right), \gamma=100\left(\mathrm{~g} /\left(\mathrm{cm}^{2} \mathrm{~s}\right)\right), \sigma_{0}=72(\mathrm{dyne} / \mathrm{cm})$, the characteristic length is $l=1(\mathrm{~cm})$. The characteristic temperature drop is chosen equal to $T_{*}=10^{0} \mathrm{C}$. We perform the calculations with different values of the coefficients $\gamma, \gamma_{0}$ of friction type "liquid - solid wall" $[8,24]$ and for two different values of static contact angle $\Phi_{0}\left(\Phi_{0}=63\right.$ and $\Phi_{0}=87$ degrees). The calculations are carried out in the case of equal values of the coefficients $\gamma$ and $\gamma_{0}\left(\gamma=\gamma_{0}=100\right.$ and $\left.\gamma=\gamma_{0}=1\right)$ under conditions of normal $\left(g=981 \mathrm{~cm} / \mathrm{s}^{2}\right)$ and low gravity $\left(g=9.81 \mathrm{~cm} / \mathrm{s}^{2}\right)$. The thermal boundary regimes have been determined according to some conditions of the physical experiments in [25]. The following values of the Nusselt number have been used $N u=\{17 ; 34\} ; T_{e x}=T_{0}$ (see (26)). The lateral boundary regime for temperature designated symbolically as $T_{s} \times T_{0}$ is considered (here $T_{s}, T_{0}$ are constant; $T_{s}>T_{0}$, see (13), (14)). The following (non-dimensional) variants are used in the calculations: $T_{s}=8, T_{0}=1$ (" $8 \times 1 ") ; T_{s}=4, T_{0}=1(" 4 \times 1 ")$. The non-dimensional parameters of the problem have the following values: $R e \approx 1112, \operatorname{Pr} \approx 7, G r \approx\{23000 ; 230\}, M a \approx 21, C a \approx 1 \cdot 10^{-5}, \bar{\alpha} \approx 0.8 \cdot 10^{-4}$, $\bar{\gamma}=1, N u \approx\{17,34\}$. The values of the Nusselt number exceed these values which correspond to the heat transfer coefficient values $\delta$ in $[25]\left(\delta \in[0,100] \mathrm{W} / \mathrm{m}^{2} \mathrm{~K}\right)$. Figures $1-4$ present the results of the model calculations in order to study the influence of the Nusselt number, defining the interface thermal regime, at external temperature $T_{e x}$ (see (12) or (26)) that coincides with lower boundary temperature $T_{0}$ on $\Gamma_{0}$.

The character of the contact angle dynamics relative to the different values of the gravity acceleration is illustrated with the help of Figures 2. We observe the increasing character of the contact angle dependence on the contact point velocity. The influence of the normal (for $g=981$ $\mathrm{cm} / \mathrm{s}^{2}$ ) and low (for $g=9.81 \mathrm{~cm} / \mathrm{s}^{2}$ ) gravity can be confirmed in the case of isothermal flow (Fig. $2(\mathrm{a})$, black lines; here the static contact angle and the friction coefficients have the values $\Phi_{0}=63$; $\gamma=\gamma_{0}=100$ ). In comparison with isothermal flow (Fig. 2(b), black solid line if $g=9.81 \mathrm{~cm} / \mathrm{s}^{2}$ and black dashed line if $g=981 \mathrm{~cm} / \mathrm{s}^{2}$ ) the thermal boundary regimes on the solid walls and free boundary have an impact on the contact angle behavior. The pictures demonstrate the similar behavior of the dynamic contact angle as in the isothermal case: increasing of the angle, when the contact point velocity increases both for the normal and low gravity. This tendency is more intensive in the case of larger values of the contact point velocity (here for $S$ larger than $\left.2\left(\mathrm{~cm} / \mathrm{s}^{2}\right)\right)$.

The numerical investigation of the contact angle dependence on the contact point velocity under conditions of normal and low gravity are carried out for the friction coefficients equal to $\gamma=\gamma_{0}=1$ (see Fig. 2(b)). The lines of the contact angle dependence are located higher in the case when $g=9.81 \mathrm{~cm} / \mathrm{s}^{2}$ than for $g=981 \mathrm{~cm} / \mathrm{s}^{2}$, see solid lines in the figures Fig. 2(b) for all the investigated regimes: for the isothermal flow (black lines), the case of " $8 \times 1$ " - thermal boundary regime on solid walls (blue lines) and the case of " $4 \times 1$ " - thermal boundary regime on solid walls (red lines). We find the especially bright differences from the isothermal regime in the case, when the $" 8 \times 1 "$ - regime is prescribed on the lateral walls. We can also speak about more intensive behavior of the dynamic contact angle in the case of larger values of the friction coefficient (here for $\gamma=100$ in comparison with $\gamma=1$ ). It is observed both for normal 


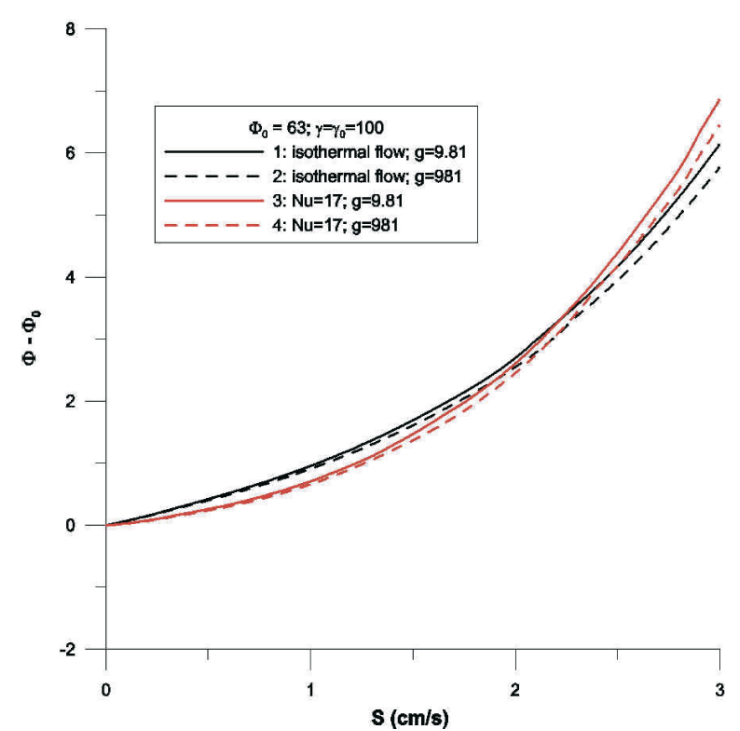

b) $\Phi_{0}=63$

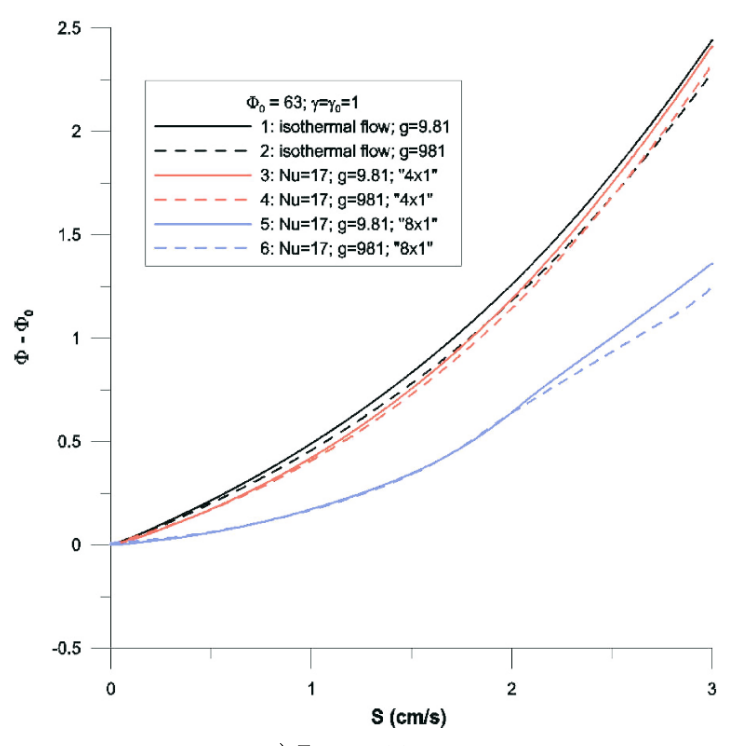

a) $\Phi_{0}=63$

Fig. 2. Dynamic contact angle. (a) Comparison of the effects of gravity acceleration. Here $\Phi_{0}=63 ; \gamma=\gamma_{0}=100$. Isothermal flow - black lines: $g=9.81$ (solid line); $g=981$ (dashed line). The regime with $N u=17$ and $" 8 \times 1 "-$ thermal boundary regime on solid walls - red lines: $g=9.81$ (solid line); $g=981$ (dashed line). (b) Comparison of the effects of gravity acceleration and the thermal boundary regimes. Here $\Phi_{0}=63 ; \gamma=\gamma_{0}=1$. Isothermal flow black lines: $g=9.81$ (solid line); $g=981$ (dashed line). The regime with $N u=17$ and "4×1" and " $8 \times 1$ " - thermal boundary regime on solid walls. The regime " $4 \times 1$ " - red lines: $g=9.81$ (solid line); $g=981$ (dashed line). The regime " $8 \times 1 "$ - blue lines: $g=9.81$ (solid line); $g=981$ (dashed line)

and low gravity (compare the black lines in Fig. 2(a) and (b) and the quantitative characteristics of the angle values; compare also the results shown with red lines in Fig. 2(a) and blue lines in Fig. 2(b)).

The topology of flow is characterized by the two-vortex flow structure that is symmetric relative to the line $y=1 / 2$ (see Fig. 3(a)). The rotor of velocity is especially intensive along the lateral walls $y=0, y=1$. In the case of higher value of the contact point velocity $(S=3 \mathrm{~cm} / \mathrm{s}$, see Fig. 3(b)) the two-vortex flow picture is characterized by a displacement of the vortex centers to the free boundary (see Fig. 1. The flow domain is characterized by the velocity field presented for the same numerical experiments data as in the Fig. 3(b)). The additional calculations on the refined grids justified the presented two-vortex flow structure with localization of the vortex centers near the free boundary.

The shapes of the free thermocapillary boundary confirm the various flow picture for the different values of the contact point velocity. The static position as well as perturbation position of the free boundary are presented in Fig. 4 in the case of two values of the static contact angle $\left(\Phi_{0}=63\right.$ and $\left.\Phi_{0}=87\right)$. The examples of the free boundary shape are presented for the values of the contact point velocity equal to $S=0$ and $S=3(\mathrm{~cm} / \mathrm{s}))$. The free boundary is symmetric relative to the line $y=1 / 2$. Especially demonstrable the free boundary behavior is presented in the case of the more high value of the contact point velocity (here equal to $3(\mathrm{~cm} / \mathrm{s})$ ) if the static contact angle is $\Phi_{0}=87$. The convexity character of the free boundary shape has been changed. 


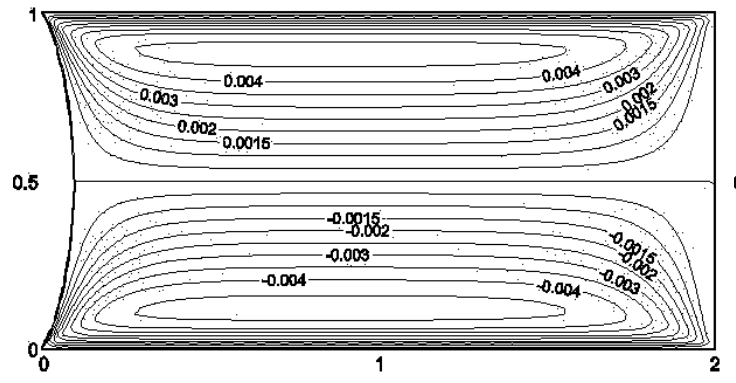

b) $S=3$

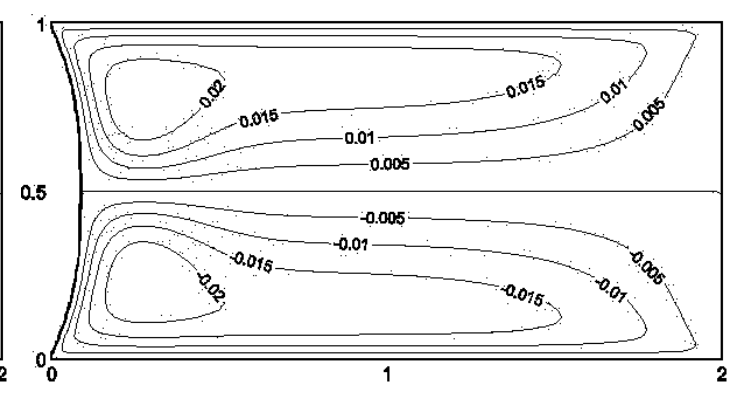

a) $S=1$

Fig. 3. Stream lines. Here $\Phi_{0}=63 ; N u=34 ; g=981 ; \gamma=\gamma_{0}=100 ; " 8 \times 1$ "- regime at solid walls

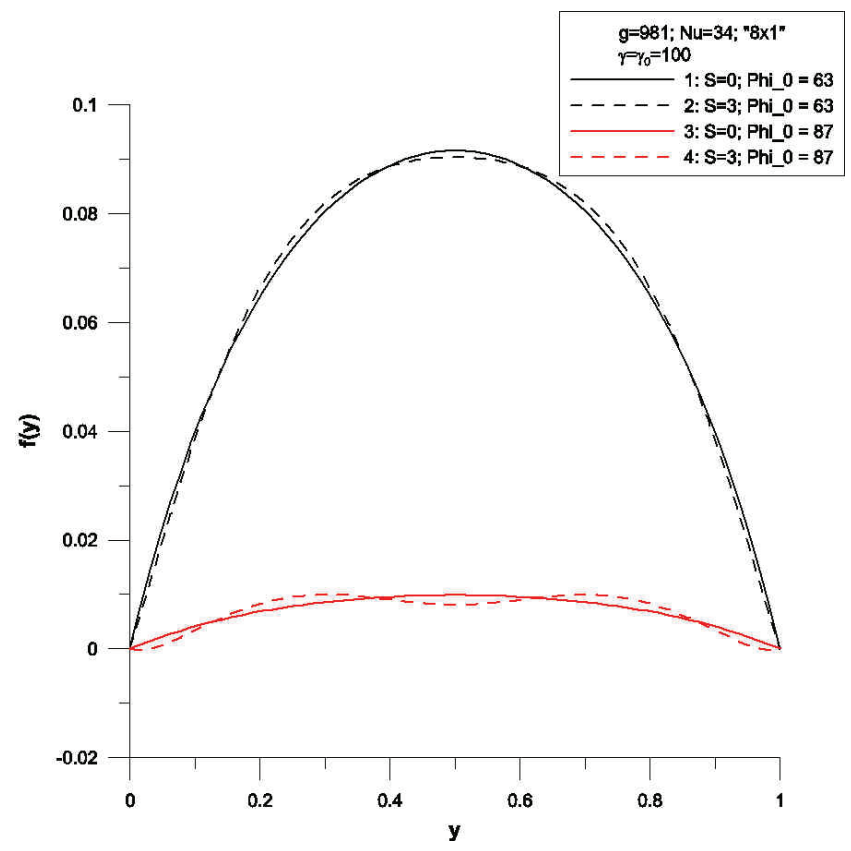

Fig. 4. Free boundary. Here $g=981 ; \gamma=\gamma_{0}=100 ; N u=34 ; " 8 \times 1$ "- regime at solid walls. $S=0$ - solid lines: black line if $\Phi_{0}=63$; red line if $\Phi_{0}=87 . S=3$ - dashed lines: black line if $\Phi_{0}=63 ;$ red line if $\Phi_{0}=87$

\section{Conclusions}

The mathematical model and the numerical algorithm are presented to study the convective fluid flows with dynamic contact angle in the case, when the contact point is moving uniformly. The increasing character of this dependence on the contact point velocity has been established. The different peculiarities of dependence of the dynamic contact angle on the contact point velocity have been investigated numerically under conditions of action of the thermal boundary regimes.

Authors gratefully acknowledge support of this work by the Russian Science Foundation (project RSF 15-19-20049). 


\section{References}

[1] D.Kröner, Aymptotische Entwicklungen für Strömungen von Flüssigkeiten mit freiem Rand und dynamischem Kontaktwinkel, Preprint 809, SFB72, Univ. Bonn, 1986.

[2] C.Baiocchi, V.V.Pukhnachov, Problems with unilateral constrains for the Navier-Stokes equations and the problem of dynamic contact angle, J. Appl. Mech. Techn. Phys., 31(1990), no. $2,185-197$.

[3] V.V.Pukhnachov, V.A.Solonnikov, On the problem of dynamic contact angle, J. Appl. Mathematics and Mechanics, 46(1982), no. 6, 771-779.

[4] Y.D.Shikhmurzaev, Mathematical modelling of wetting hydrodynamics, Fluid Dyn. Res., 13(1994), 45-64.

[5] V.A.Solonnikov, Solvability of a problem on the plain motion of a heavy viscous incompressible capillary liquid partially filling a container, Math. USSR, Izv., 14(1980), 193-221.

[6] V.S. Ajaev, G.M. Homsy, Modeling shapes and dynamics of confined bubbles, Annu. Rev. Fluid Mech., 38(2006), 277-307.

[7] D.Kröner, The flow of a fluid with a free boundary and dynamic contact angle, ZAMM, 67(1990), 304-306.

[8] W.Doerfler, O.Goncharova, D.Kroener, Fluid flow with dynamic contact angle: numerical simulation, ZAMM, 82(2002), no. 3, 167-176.

[9] D.Kröner, Asymptotic expansion for a flow with a dynamic contact angle, In: Heywood J., Masuda K., Rautmann R., Solonnikov V.A. (eds.): The Navier-Stokes equations theory and numerical methods, Proc. Conf, Oberwolfach. Lect. Notes Math. 1431, Springer Berlin, 1990, 49-59.

[10] D. Kröner, W. M. Zajaczkowski, Free boundary flow with dynamic contact angle in 2D, Part 1, Existence for the linearized problem, Manuscript, Univ. Freiburg, 2000.

[11] D.Kröner, W.M.Zajaczkowski, Free boundary flow with dynamic contact angle in 2D, Part 2, Existence, Manuscript, Univ. Freiburg, 2000.

[12] D. Joseph, Stability of fluid motions, Springer Verlag, Berlin, Heidelberg, New York, 1976.

[13] V.K.Andreev, Yu.A.Gaponenko, O.N.Goncharova, V.V.Pukhnachov, Mathematical models of convection, Berlin-Boston, Walter de Gruyter, 2012.

[14] V.V.Pukhnachov, Viscous fluid flow with free boundaries, Novosibirskii Gos. Universitet, Novosibirsk, 1989 (in Russian).

[15] A.S. Ovcharova, Method of calculating steady-state flows of a viscous fluid with free boundary in vortex-stream function variables, J. of Appl. Mech. Techn. Phys., 39(1998), no. 2, $211-219$.

[16] Goncharova, O.N., Pukhnachov, V.V., Kabov, O.A., Solutions of special type describing the three dimensional thermocapillary flows with an interface, Int. Journal of Heat and Mass Transfer, 54(5)(2012) 715-725. 
[17] J.Douglas jr., J.E.Gunn, A general formulation of alternating direction methods, I, Parabolic and hyperbolic problems, Numer. Math., 6(1964) 428-453.

[18] N.N. Yanenko, The method of fractional steps: the solution of problems of mathematical physics of several variables, Springer Verlag, Berlin, Heidelberg, New York, 1971.

[19] P.J.Roache, Computational Fluid Dynamics, Hermosa Publishers, Albuquerque, 1976.

[20] G.I.Marchuk, Splitting methods, Nauka, Moscow, 1988 (in Russian).

[21] A.A.Samarsky, E.S.Nikolaev Methods of solution of the grid equations, Nauka, Moscow, 1978 (in Russian).

[22] Physical Values, Handbook, Moscow, Energoatomizdat Publisher House, 1991 (in Russian).

[23] N.B. Vargaftik et al., Handbook of thermal conductivity of liquids and gases, CRC Press, 1994

[24] E.B.Gutoff, C.E.Kendrick, Dynamic contact angles. AIChE J., 28(1982) 459-466.

[25] E.Ya.Gatapova, A.A.Semenov, D.V.Zaitsev, O.A.Kabov, Evaporation of a sessile water drop on a heated surface with controlled wettability, Colloids and surfaces A, Psysicochem. Eng. Aspects, 441(2014), 776-785.

\title{
Численное исследование зависимости динамического контактного угла от скорости движения точки контакта в задаче о конвективном движении жидкости
}

Ольга Н. Гончарова

Алтайский государственный университет Ленина, 61, Барнаул, 656049

Россия

Алла В. Закурдаева Институт теплофизики СО РАН Ак. Лаврентьева, 1, Новосибирск, 630090

Россия

\begin{abstract}
Изучается задача движения жидкости с динамическим контактным углом в случае равномерно движущейся точки контакта. Математическое моделирование проводится на основе аппроксимации Обербека-Буссинеска уравнений Навъе-Стокса. На термокапиллярной свободной границе выполняются кинематическое, динамическое условия и условие теплового обмена с внешней средой третъего рода. Условия прилипания выполняются на твердых границах, которые поддерживаются при постоянной температуре. Данные условия представляют собой условия пропорциональности касательных напряжений разнице касателъных скоростей жидкости и твердой стенки. Численно исследуется зависимость динамического контактного угла от скорости движения точки контакта. Результаты демонстрируют поведение динамического контактного угла и различия в характеристиках течения в зависимости от различных значений скорости движения точки контакта, коэффициентов трения, ускорения силь тяжести и интенсивности граничного теплового режима.
\end{abstract}

Ключевые слова: конвективное течение, свободная граница, динамический контактный угол, движущаяся точка контакта, математическая модель, вычислительный алгоритм. 